\title{
Comparison of the Dietary Intake Loss Between Total and Distal Gastrectomy for Gastric Cancer
}

\author{
MASATO NAKAZONO ${ }^{1,2^{*}}$, TORU AOYAMA ${ }^{1,2^{*}}$, TSUTOMU HAYASHI ${ }^{1}$, KENTARO HARA $^{1}$, \\ KENKI SEGAMI $^{1,2}$, YOTA SHIMODA ${ }^{1}$, SHINSUKE NAGASAWA ${ }^{1}$, YUTA KUMAZU $^{1}$, TAKANOBU YAMADA ${ }^{1}$, \\ HIROSHI TAMAGAWA ${ }^{2}$, MANABU SHIOZAWA ${ }^{1}$, SOICHIRO MORINAGA ${ }^{1}$, YASUSHI RINO ${ }^{2}$, \\ MUNETAKA MASUDA $^{2}$, TAKASHI OGATA ${ }^{1}$ and TAKASHI OSHIMA ${ }^{1}$ \\ ${ }^{1}$ Department of Gastrointestinal Surgery, Kanagawa Cancer Center, Yokohama, Japan; \\ ${ }^{2}$ Department of Surgery, Yokohama City University, Yokohama, Japan
}

\begin{abstract}
Background/Aim: The changes of dietary intake (DI) after gastrectomy have not been objectively reported. It has not been clear how much DI loss is experienced after total gastrectomy (TG) in comparison to after distal gastrectomy (DG). This study quantified the changes of DI after gastrectomy, and clarified how much DI loss is experienced after TG. Patients and Methods: This was a prospective observational study. Patients who underwent gastrectomy for gastric cancer were enrolled. The DI loss was evaluated at 1 and 3 months postoperatively. Results: Thirty-three patients underwent TG, and 117 patients underwent DG. The median \%DI loss of the overall study population at 1 and 3 months after surgery was $-9.3 \%$ and $-3.6 \%$. The median \%DI loss at 1 and 3 months postoperatively was $-15.6 \%$ and $-5.3 \%$ in TG group, $-8.9 \%$ and $-3.3 \%$ in DG group ( $p=0.10$ and 0.49 , respectively). Conclusion: The patients experienced DI loss of approximately $10 \%$ at 1 month after gastrectomy. Patients who received TG tended to show a greater \%DI loss at 1 month postoperatively.
\end{abstract}

Gastrectomy is an essential treatment for gastric cancer (1-3). Body weight loss is a common problem after gastrectomy for gastric cancer (4). In particular, after total gastrectomy (TG), patients often lose $>10 \%$ of their preoperative body weight

This article is freely accessible online.

*These Authors contributed equally to this article.

Correspondence to: Toru Aoyama, Department of Gastrointestinal Surgery, Kanagawa Cancer Center, 2-3-2 Nakao, Asahi-ku, Yokohama 241-8515, Japan. Tel: +81 0455202222, e-mail: taoyama@lilac.plala.or.jp

Key Words: Gastric cancer, dietary intake, gastrectomy, body weight, nutrition status.
$(5,6)$. The postoperative body weight loss at 1 month after surgery affects compliance with adjuvant chemotherapy and patient survival $(7,8)$. Thus, it is necessary to prevent body weight loss in patients after gastrectomy. Various factors that possibly underlie the body weight loss after gastrectomy have been considered, including decreased dietary intake due to a loss of reservoir function, malabsorption, hyper-catabolism due to surgical stress, lack of exercise, reduction in the blood ghrelin levels, and others (9-13). A decreased dietary intake has been considered a predominant factor in the body weight loss after gastrectomy $(12,13)$. However, the changes in dietary intake after gastrectomy have not been objectively reported. Moreover, patients who undergo TG are reported to experience much more body weight loss in comparison to those who undergo distal gastrectomy (DG). It has not been clear how much dietary intake loss was experienced after TG in comparison to after DG.

The Food Frequency Questionnaire with 82 food items (FFQW82) is a self-administered questionnaire that inquiries about the intake frequency and portion size over a period of 1 month from a list of foods with pictures $(14,15)$. In comparison to a previous tool that calculates the dietary intake, the FFQW tool has certain clinical benefits. First, patients can easily answer the FFQW82 by choosing food items, portion size, and intake frequency from a questionnaire with pictures. FFQW82 has been used for the nutritional education of adolescents of 12-13 years of age (14). Thus, we considered that elderly patients could easily answer the FFQW82. Second, using the FFQW82, the energy and nutritional intake for breakfast, lunch, and dinner, as well as for the whole day can be estimated. After gastrectomy, it is recommended that patients decrease the portion size of each meal, and increase their intake frequency (16). In nutritional counselling after gastrectomy, it is an advantage to know not only the intake status for the whole day but also for each meal. Thus, the FFQW82 is suitable for calculating the dietary intake after gastrectomy 
Table I. Comparison of the patient background.

\begin{tabular}{|c|c|c|c|}
\hline & TG $(n=33)$ & DG $(n=117)$ & $p$-Value \\
\hline Age, years (median) (range) & $67(45-83)$ & $66(27-86)$ & 0.33 \\
\hline Gender & & & 0.01 \\
\hline Male & $27(82 \%)$ & $68(58 \%)$ & \\
\hline Female & $6(18 \%)$ & $49(42 \%)$ & \\
\hline Height, $\mathrm{cm}$ (median) (range) & $\begin{array}{c}163.6 \\
(147-175.6)\end{array}$ & $\begin{array}{c}162 \\
(132.8-182.8)\end{array}$ & 0.97 \\
\hline $\begin{array}{l}\text { Body weight, } \mathrm{kg} \\
\text { (median) (range) }\end{array}$ & $\begin{array}{c}60.3 \\
(36.9-73.0)\end{array}$ & $\begin{array}{c}58.5 \\
(35.3-88.2)\end{array}$ & 0.54 \\
\hline ASA-PS & & & 0.45 \\
\hline 1 & $8(24 \%)$ & $40(34 \%)$ & \\
\hline 2 & $25(76 \%)$ & $74(63 \%)$ & \\
\hline 3 & 0 & $3(3 \%)$ & \\
\hline
\end{tabular}

ASA-PS, American Society of Anesthesiologists physical status; TG, total gastrectomy; DG, distal gastrectomy.

The primary aim of the present study was to quantify the changes of dietary intake after gastrectomy, using the FFQW82. The second aim was to clarify how much dietary intake loss was experienced after TG in comparison to after DG.

\section{Patients and Methods}

Patients. This prospective observational study was conducted from May 2011 to November 2014 at the Kanagawa Cancer Center. The eligibility criteria were as follows: 1) histologically proven gastric adenocarcinoma diagnosed as pathological stage IA or IB according to the Japanese Classification of Gastric Carcinoma (17); 2) patients receiving nutritional counselling 2-4 days before surgery and 1 month and 3 months postoperatively. Patients who received preoperative or postoperative chemotherapy were excluded. The patients were classified into those who received total gastrectomy (the TG group) and those who received distal gastrectomy (the DG group).

Perioperative care. All patients received perioperative care using the enhanced recovery after surgery (ERAS) protocol. The details of this protocol have been reported in a previous study $(18,19)$. In brief, patients could eat a normal diet until dinner on the day before surgery, and could drink oral rehydration solution until $3 \mathrm{~h}$ before surgery. Premedication was not administered. The nasogastric tube was removed immediately after surgery. Oral intake was initiated on postoperative day (POD) 2, beginning with water and an oral nutritional supplement. The patients began soft diet intake on POD 3 and were stepped up to regular food every 2 days ( 3 steps). The patients were discharged when they had successfully achieved adequate pain relief and soft food intake, had returned to their preoperative mobility level, and exhibited normal laboratory data on POD7.

Study schedule. The patients received perioperative nutritional counselling on the day of hospitalization, the day of discharge, and 1 month and 3 months postoperatively. In counselling during
Table II. The surgical and pathological outcomes.

\begin{tabular}{|c|c|c|c|}
\hline & TG $(n=33)$ & DG $(n=117)$ & $p$-Value \\
\hline Approach & & & 0.36 \\
\hline Laparotomy & $9(27 \%)$ & $42(36 \%)$ & \\
\hline Laparoscopic surgery & $24(73 \%)$ & $75(64 \%)$ & \\
\hline Lymph node dissection & & & 0.69 \\
\hline D1+ dissection & $27(82 \%)$ & $99(85 \%)$ & \\
\hline D2 dissection & $6(18 \%)$ & $18(15 \%)$ & \\
\hline Reconstruction & & $<0.01$ & \\
\hline Billroth-I & $0(0 \%)$ & $94(80 \%)$ & \\
\hline Roux-en-Y & $33(100 \%)$ & $23(20 \%)$ & \\
\hline $\begin{array}{l}\text { Operation time }, \text { min } \\
\text { (median) (range) }\end{array}$ & $\begin{array}{c}309 \\
(145-435)\end{array}$ & $\begin{array}{c}262 \\
(85-512)\end{array}$ & 0.01 \\
\hline Bleeding, g (median) (range) & $75(5-910)$ & $60(0-950)$ & 0.075 \\
\hline Postoperative complications & 0.25 & & \\
\hline$(+)$ & $7(21 \%)$ & $14(11 \%)$ & \\
\hline$(-)$ & $26(79 \%)$ & $103(89 \%)$ & \\
\hline $\begin{array}{l}\text { Duration of hospitalization, } \\
\text { day (median) (range) }\end{array}$ & $9(6-25)$ & $8(7-58)$ & 0.22 \\
\hline Pathological $\mathrm{T}$ factor & & & 0.53 \\
\hline T0-1 & $29(88 \%)$ & $107(91 \%)$ & \\
\hline $\mathrm{T} 2$ & $4(12 \%)$ & $10(9 \%)$ & \\
\hline Pathological $\mathrm{N}$ factor & & & 0.46 \\
\hline No & $32(97 \%)$ & $107(91 \%)$ & \\
\hline N1 & $1(3 \%)$ & $10(9 \%)$ & \\
\hline Pathological stage & & & 0.79 \\
\hline IA & $28(85 \%)$ & $97(83 \%)$ & \\
\hline IB & $5(15 \%)$ & $20(17 \%)$ & \\
\hline
\end{tabular}

TG, Total gastrectomy; DG, distal gastrectomy.

hospitalization, -which was 2-4 days before surgery, nutritionists analyzed patients' preoperative dietary intake conditions and measured their body weight. In counselling on discharge, nutritionists provided education on how to eat meals after gastrectomy, including (but not limited to): to increase intake frequency; to decrease the portion size in each meal; to chew frequently; to eat slowly. In counseling at 1 and 3 months postoperatively, nutritionists analyzed the patients' postoperative dietary intake conditions, measured their body weight, and provided education on how the patients could improve their nutritional status based on the results.

Analysis of the dietary intake. The dietary intake was evaluated in perioperative nutritional counselling using the FFQW82. The FFQW82 was established and validated by Watanabe et al. in 2011 (14). The FFQW82 is a self-administered questionnaire that is designed to present 82 food items according to 16 food groups and inquire about the intake frequency and portion size of each meal. The intake frequency is indicated by six categories: $(0=$ 'absolutely do not eat'; $1=$ 'eat once or twice per month'; $2=$ "eat once or twice per week'; $3=$ 'eat 3 to 4 times per week'; $4=$ 'eat 5 to 6 times per week'; $5=$ 'eat everyday'). The portion size is described as 'small', 'medium', or 'large'. The standard amount for 'medium' is shown by size on the food list with pictures. 'Small' is defined as half the amount of 'medium', whereas 'large' is defined as 1.5 times the amount of 'medium'. Patients completed the FFQW82 in 


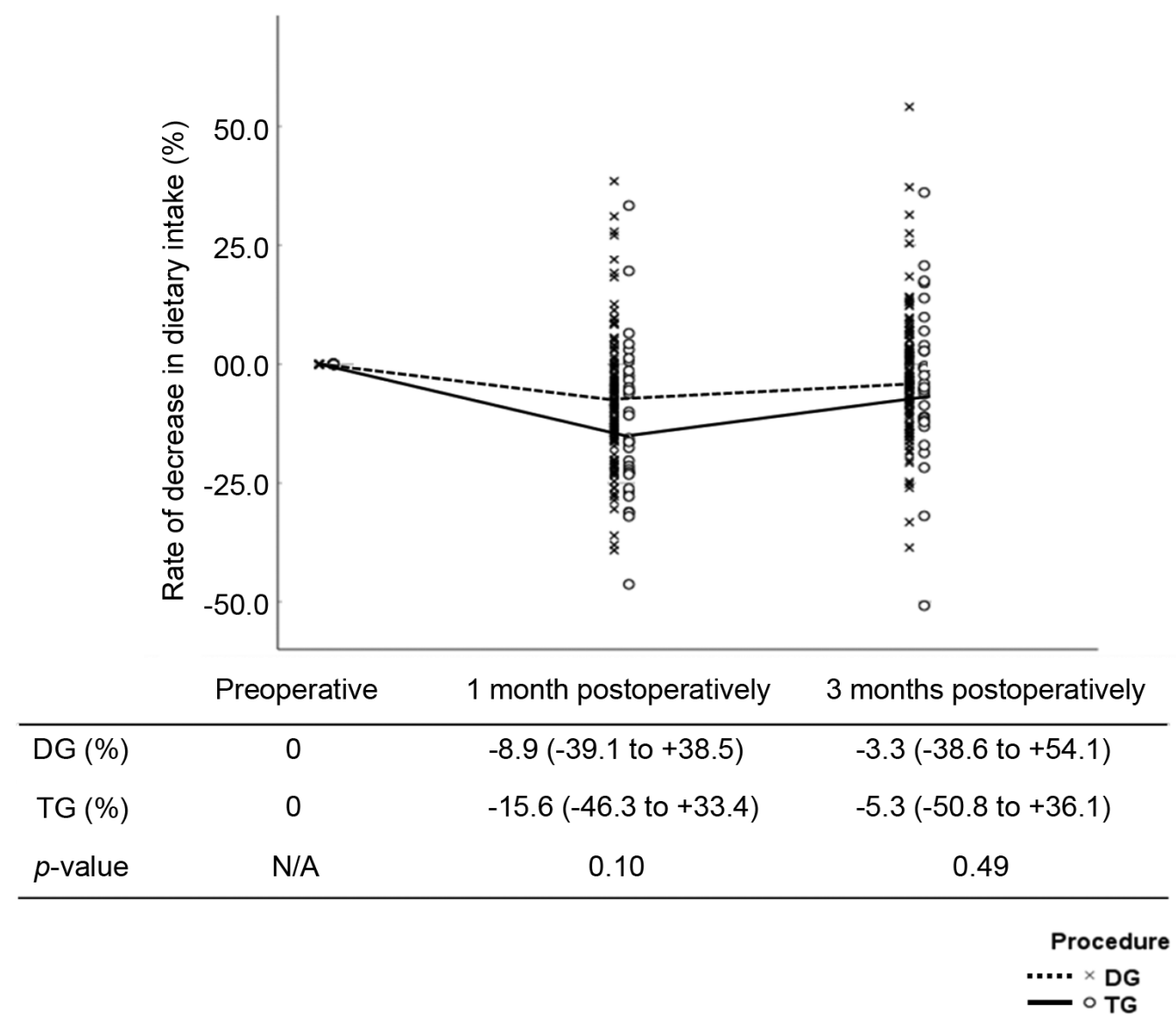

Figure 1. Rate of decrease in dietary intake (\%) in the total gastrectomy (TG) and distal gastrectomy (DG) groups.

approximately $30 \mathrm{~min}$. Based on the responses to the FFQW82, the estimated intake of energy and several nutrients for a whole day were calculated by simply summing up the estimated intake of food items for each meal. Microsoft ${ }^{\circledR}$ Excel (Microsoft Inc., Redmond, WA, USA) was used to obtain the nutrient composition for items on the FFQW82.

Evaluations, statistical analyses, and ethics. Dietary intake (DI) loss was defined as $\%$ DI loss=(DI at 1 month postoperatively and 3 months postoperatively - preoperative DI) $\times 100$ /preoperative DI. Body weight (BW) loss was defined as $\% \mathrm{BW}$ loss=(BW at postoperative 1 month and 3 months postoperatively - preoperative $\mathrm{BW}) \times 100 /$ preoperative $\mathrm{BW}$. The values were expressed as the median and range. The data were compared between the TG and DG groups using the Chi-squared test and the Mann-Whitney $U$-test. The correlation coefficients between the \%DI loss and \%BW loss were calculated using Spearman's rank correlation coefficient. $p$ Values of $<0.05$ were considered to indicate statistical significance. Analyses were performed using SPSS version 25.0 (Statistical Package for the Social Science; SPSS, Chicago, IL, USA). This study was approved by the Institutional Review Board (IRB) of Kanagawa Cancer Center (2018 epidemiologic study-20). The study was conducted in accordance with the Declaration of Helsinki. All subjects signed consent forms.

\section{Results}

Patient characteristics. A total of 150 patients were examined in this study. Thirty-three patients underwent TG, and 117 underwent DG. The median age (range) of the 150 patients was 67 (27-86) years. One hundred-two patients $(68 \%)$ had comorbidities, including hypertension $(n=61$; $41 \%)$, diabetes mellitus $(n=16 ; 11 \%)$, and chronic obstructive pulmonary disease $(n=11 ; 7 \%)$. The patient characteristics are shown in Table I. There was a statistically significant difference in the sex ratio of the two groups. The TG group included a higher percentage of male patients than the DG group (TG $82 \%$ vs. DG $58 \%, p=0.01$ ). The other characteristics of the two groups were similar.

Surgical and pathological outcomes. In the DG group, 94 patients underwent Billroth I reconstruction, and 23 patients underwent Roux-en-Y reconstruction. The approach, lymph node dissection, postoperative complications, and duration of hospitalization were similar between the two groups. The operative time in the TG group was significantly longer than 


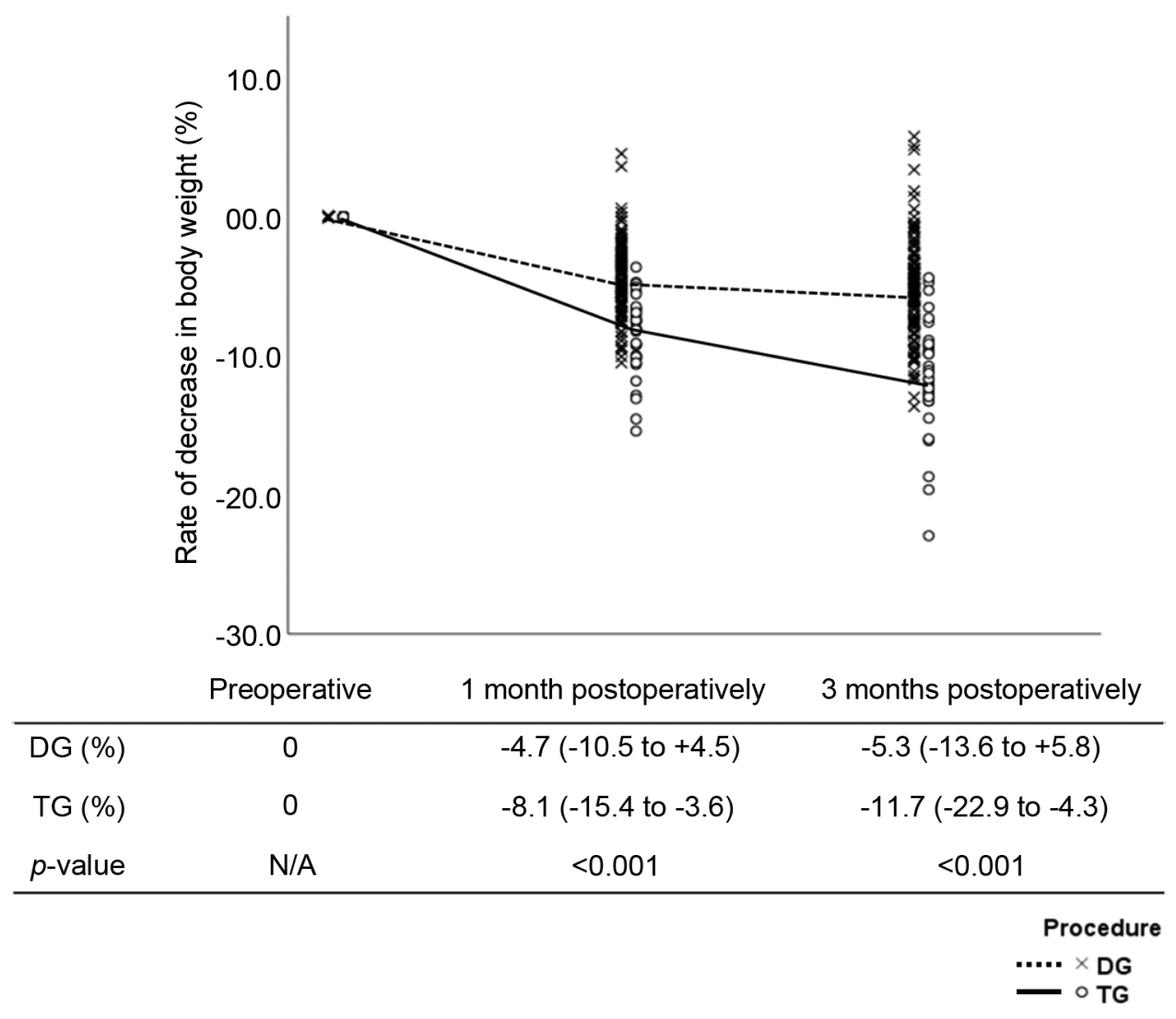

Figure 2. Rate of decrease in body weight (\%) in the total gastrectomy (TG) and distal gastrectomy (DG) groups.

that in the DG group (TG $309 \min v s$. DG $262 \mathrm{~min}, p=0.01$ ). Although not significant, patients in the TG group tended to experience more bleeding than those in the DG group (TG $75 \mathrm{ml} v s$. DG $60 \mathrm{ml}, p=0.075)$. The pathological outcomes of the two groups did not differ to a statistically significant extent (Table II).

Dietary intake and body weight changes. The median DI (range) of the overall population before surgery, at 1 month after surgery, and at 3 months after surgery, was 1,713.5 $\mathrm{kcal} / \mathrm{day},(1,126-2,330), 1541.5 \mathrm{kcal} / \mathrm{day},(986-2,195)$, and $1638.5 \mathrm{kcal} /$ day $(816-2,443)$, respectively. The median DI (range) before surgery was $1794 \mathrm{kcal} /$ day $(1,263-2,118)$ in the TG group, and $1674 \mathrm{kcal} /$ day $(1,126-2,330)$ in the DG group ( $p=0.03)$. The median DI (range) at 1 month after surgery was $1520 \mathrm{kcal} /$ day $(1,018-2,071)$ in the TG group, and $1543 \mathrm{kcal} /$ day $(986-2,195)$ in the DG group $(p=0.98)$. The median DI (range) at 3 months after surgery was 1692 $\mathrm{kcal} /$ day $(816-2,013)$ in the TG group, and $1624 \mathrm{kcal} /$ day $(917-2,443)$ in the DG group $(p=0.26)$.

The median \%DI loss (range) of the overall study population at 1 month and 3 months after surgery was $-9.3 \%$ ( -46.3 to
$38.5)$ and $-3.6 \%$ (-50.8 to 54.1$)$. The median \%DI loss (range) at 1 month postoperatively was $-15.6 \%$ ( -46.3 to 33.4$)$ in the TG group, and $-8.9 \%(-39.1$ to 38.5$)$ in the DG group $(p=0.10)$. The median $\%$ DI loss at 3 months postoperatively was $-5.3 \%$ ( -50.8 to 36.1$)$ in the TG group, and $-3.3 \%(-38.6$ to 54.1$)$ in the DG group $(p=0.49)$. The $\%$ DI loss after gastrectomy at 1 month postoperatively did not differ to a statistically significant extent in the TG and DG groups; however, the patients in the TG group tended to experience greater \% DI loss in comparison to the DG group (Figure 1).

The median $\% \mathrm{BW}$ loss (range) of 1 month postoperatively was $-8.1 \%(-15.4$ to -3.6$)$ in the TG group, and $-4.7 \%$ (10.5 to 4.5$)$ in the DG group $(p<0.01)$. The median $\% \mathrm{BW}$ loss at 3 months postoperatively was $-11.7 \%(-22.9$ to -4.3$)$ in the TG group, and $-5.3 \%$ ( -13.6 to 5.8 ) in the DG group $(p<0.01)$. The \%BW loss of the two groups showed statistically significant differences at 1 month and 3 months postoperatively (Figure 2).

The DI and BW were compared at the preoperative examination. The correlation coefficients of the overall population, the TG group, and the DG group were 0.395 $(p<0.001), 0.417(p=0.016)$, and $0.451(p<0.001)$, respectively 


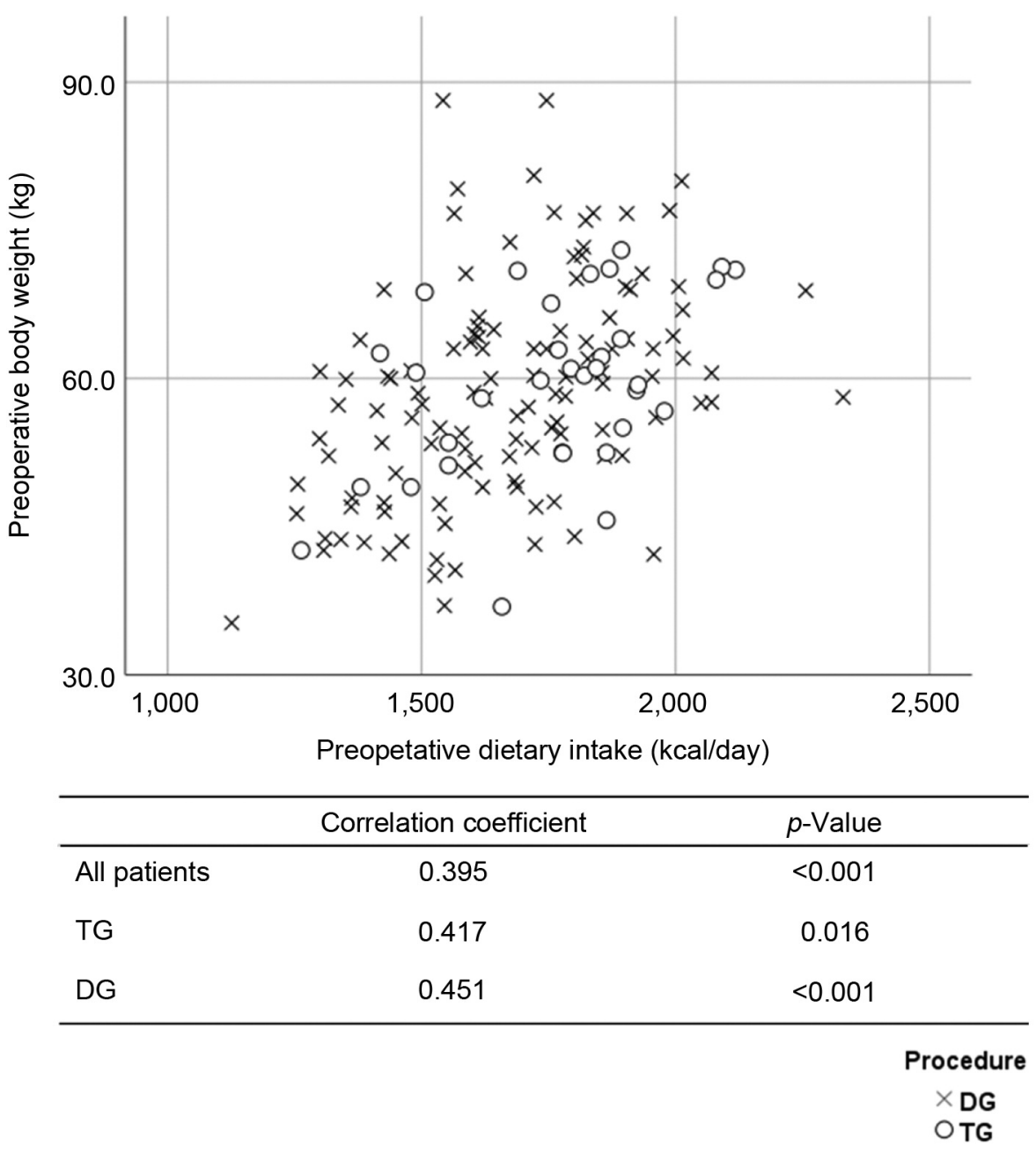

Figure 3. Scatter plot between the dietary intake and the body weight before surgery.

(Figure 3 ). The $\%$ DI loss at 1 month postoperatively and the $\% \mathrm{BW}$ loss at 1 month postoperatively were compared. The correlation coefficients of the overall study population, the TG group, and the DG group were $0.218(p=0.007), 0.407$ $(p=0.019)$, and $0.142(p=0.125)$, respectively (Figure 4$)$. The $\% \mathrm{DI}$ loss at 1 month postoperatively and the \% BW loss at 3 months postoperatively were compared. The correlation coefficients of the overall study population, the TG group, and the DG group were $0.280(p=0.001), 0.332(p=0.059)$, and $0.254(p=0.006)$, respectively (Figure 5). The \%DI loss at 3 months postoperatively and the $\% \mathrm{BW}$ loss at 3 months postoperatively were compared. The correlation coefficients of the overall study population, the TG group, and the DG group were $0.280(p=0.001), 0.071 \quad(p=0.695)$, and 0.189 $(p=0.042)$, respectively. After TG, the \%DI loss at 1 month postoperatively was correlated with the $\% \mathrm{BW}$ loss at 1 month postoperatively, and 3 months postoperatively.

\section{Discussion}

The aim of the study was to quantify the changes in dietary intake after gastrectomy, and to clarify how much dietary intake loss was experienced after TG. The major finding was that patients experienced DI loss of approximately $10 \%$ of the preoperative DI at 1 month after gastrectomy. The \%DI loss at 1 month postoperatively was correlated with the $\% \mathrm{BW}$ loss at 1 month postoperatively, and 3 months postoperatively. In addition, the \%DI loss after gastrectomy in the TG and DG did not differ to a statistically significant extent at 1 month postoperatively, while at 1 month postoperatively, the patients in the TG group tended to experience greater \%DI loss in comparison to the DG group. After TG, the \%DI loss at 1 month postoperatively was found to be correlated with the $\% \mathrm{BW}$ loss at 1 month postoperatively, and 3 months postoperatively. Taken together, our results suggested that if the 


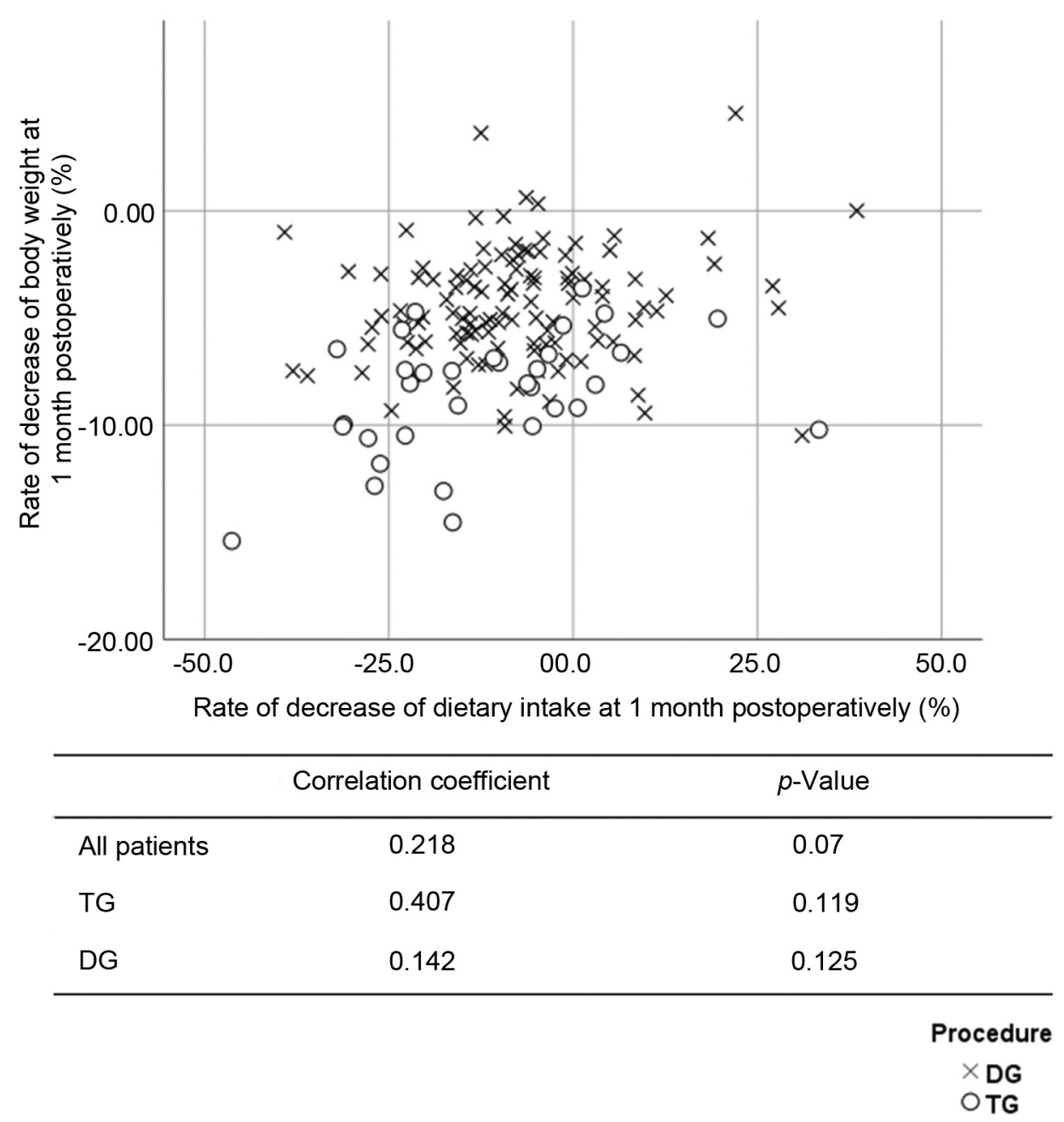

Figure 4. Scatter plot between the rate of decrease of dietary intake at 1 month postoperatively and the rate of decrease of body weight at 1 month postoperatively.

DI loss at 1 month postoperatively could be prevented by nutritional intervention until 1 month after surgery, the BW loss after gastrectomy might be suppressed. In particular, after TG, nutritional intervention until 1 month after surgery was expected to have a greater preventive effect in relation to BW loss.

The present study demonstrated that DI decreased by approximately $10 \%$ from $1,713.5 \mathrm{kcal} /$ day before surgery to $1,541.5 \mathrm{kcal} /$ day at 1 month postoperatively, and that the DI decreased by approximately $4 \%$ from $1,713.5 \mathrm{kcal} /$ day before surgery to $1,638.5 \mathrm{kcal} /$ day at 3 months postoperatively. Previously, several studies quantified the changes in DI after gastrectomy $(20,21)$. Lee et al. examined the effects of intensive education after gastrectomy for early gastric cancer on the nutritional status and diet quality, and demonstrated that the intake of patients at one week post-discharge was $1,390.5 \mathrm{kcal} / \mathrm{day}$, while that at 3month post-discharge was $1,725.6 \mathrm{kcal} /$ day (20). Noguchi et al. examined the nutritional alternation after gastrectomy, regarding caloric intake and the utilization of nutrients. In their study, at the time of discharge, the DI after TG was $1,020 \mathrm{kcal} / \mathrm{day}$, and the DI after subtotal gastrectomy was $1,326 \mathrm{kcal} / \mathrm{day}$ (22). There are several possible reasons to why the DI at 1 month after surgery in the present study was higher in comparison to previous studies (20). The first possible reason must be differences in perioperative management, which was based on the ERAS protocol in the present study. The postoperative period of "nothing by mouth" and the length of hospital stay were shorter in comparison to previous studies $(20,22)$. The ERAS protocol was expected to reduce DI loss at 1 month postoperatively. The second possible reason is the patients' background and operative details, which included progression classification, surgical procedure, method of reconstruction, and other factors. In the present study, we restricted enrollment to 


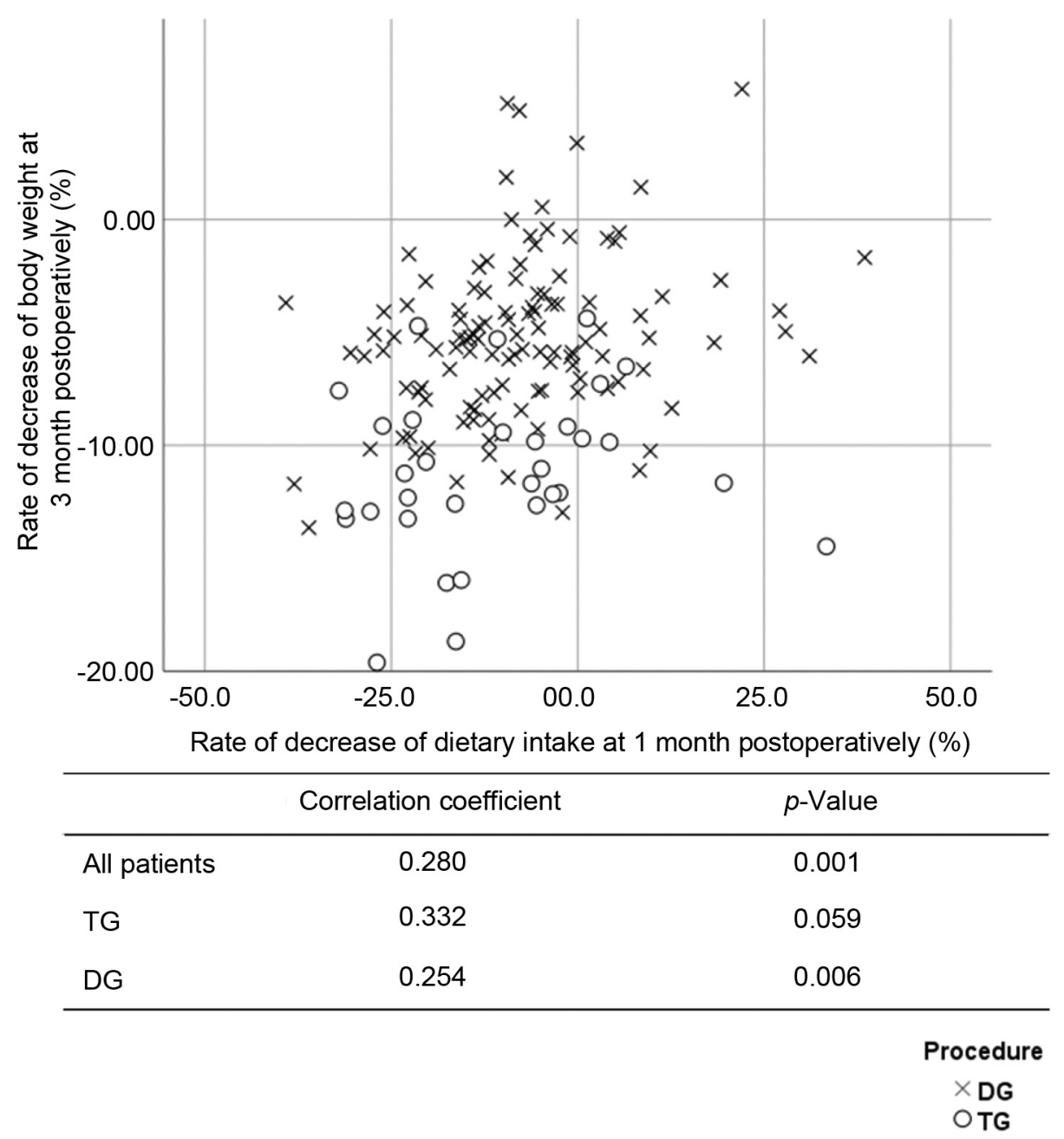

Figure 5. Scatter plot between the rate of decrease of dietary intake at 1 month postoperatively and the rate of decrease of body weight at 3 months postoperatively.

patients with pathological stage IA or IB, in order to eliminate the influence of symptoms caused by cancer and postoperative adjuvant chemotherapy. The third possible reason is the method used to calculate DI. In the present study, DI was calculated using the FFQW82, which is expected to reflect the DI over a one-month period. In previous studies, the DI was calculated based on the patient's description of the daily food intake over three consecutive days. Moreover, the preoperative DI was not described in the previous studies, and the rate of DI loss from before surgery to 1 month postoperatively was not calculated. Taken together, it was considered that the present study could quantify the change in DI after gastrectomy more accurately in comparison to previous studies. In the present study, the DI loss at 1 month postoperatively was lower in comparison to previous studies. However, the \%DI loss at 1 month postoperatively was still found to be approximately $10 \%$, and was correlated with the $\% \mathrm{BW}$ loss at 1 month postoperatively, and 3 months postoperatively. Therefore, the changes in DI at 1 month postoperatively should be targeted in order to suppress the BW loss after gastrectomy.

The second aim of the present study was to clarify how much DI loss was experienced after TG, in comparison to after DG. In the present study, the \%DI loss after gastrectomy in the TG and DG groups did not differ to a statistically significant extent at 1 month after surgery, while the patients in the TG group tended to experience greater \%DI loss in comparison to the DG group (TG $-15.6 \%$ vs. DG $-8.9 \%$, $p=0.10$ ). From 1 month after surgery to 3 months after surgery, the patients of both groups showed a recovery in \%DI loss. At 3 months postoperatively, the \%DI loss of the two groups did not differ to a statistically significant extent (TG $-5.3 \% \mathrm{vs}$. DG $-3.3 \%, p=0.49)$. In contrast, the $\% \mathrm{BW}$ loss of the TG group was significantly greater than that of the DG group at 
1 month (TG $-8.1 \%$ vs. DG $-4.7 \%, p<0.01$ ), and 3 months (TG $-11.7 \%$ vs. DG $-5.3 \%, p<0.01$ ) postoperatively. Furthermore, from 1 to 3 months after surgery, the $\% \mathrm{BW}$ loss of the TG group continued to decrease, while the $\% \mathrm{BW}$ loss after DG tended to stop decreasing. The patients of both groups showed similar changes in \%DI loss, which decreased at 1 month postoperatively, and recovered at 3 months postoperatively. However, the changes in \% BW loss differed between the two groups. The $\% \mathrm{BW}$ loss after TG continued to decrease at 1 month and 3 months postoperatively. The $\%$ BW loss after DG decreased at 1 month postoperatively and stopped decreasing at 3 months postoperatively. There are two possible explanations for the differences between the \%DI loss and the $\% \mathrm{BW}$ loss. The first possible reason is that the DI loss after TG at 1 month surgery affected the BW loss at both 1 month and 3 months after surgery. The second possible reason is that the BW loss after TG were more affected by additional factors, other than DI loss after DG. Other factors that were considered included malabsorption and hyper-catabolism; however, these could not be identified based on the results of the present study $(9-11,21)$. Accordingly, in order to suppress the BW loss after TG, more intensive nutritional intervention should be provided until 1 month after surgery, and other factors should be investigated and improved.

The present study is associated with certain limitations. First, this study was a prospective single-center study with a small sample size. The number of the patients may be too small to lead to definite conclusions. The results need to be confirmed in another cohort or in a prospective multicenterstudy. Second, the DI was quantified using FFQW82 which could not directly measure the DI. This is the weakness of using FFQW82. However, as described above, the present study using the FFQW82 was considered to quantify the change in DI after gastrectomy more accurately in comparison to previous studies. The strengths of using FFQW82 are the ease to answer and a lot of information which includes the energy and nutritional intake for breakfast, lunch, and dinner, as well as for the whole day. However, the most suitable methods for calculating the DI after gastrectomy should be investigated. Third, the patients in this study showed good compliance, as they accurately completed the FFQW82 three times at (before surgery, 1 month postoperatively, and 3 months postoperatively). Thus, it is possible that there was a selection bias.

In conclusion, at 1 month after gastrectomy, the patients showed approximately $10 \%$ DI loss. The comparison of the TG and DG groups revealed that, at 1 month postoperatively, patients who received TG tended to experience greater \%DI in comparison to those who received DG. After TG, the \%DI loss at 1 month postoperatively was found to be correlated with the \%BW loss at 1 month and 3 months postoperatively. To suppress the BW loss after TG, more intensive nutritional intervention should be provided until 1 month after surgery.

\section{Conflicts of Interest}

The Authors declare no competing interests in association with the present study.

\section{Authors' Contributions}

MN, TA, and TH made substantial contributions to the conception and design. MN, TA, TH, KH, YS, SN, KS, YK, TY, TO1 (Takashi Ogata), and TO2 (Takashi Oshima) made substantial contributions to the acquisition of data or the analysis and interpretation of data. MN, TA, TH, KH, YS, SN, YK, TY, HT, MS, SM, YR, MM, TO1, and TO2 were involved in drafting the manuscript or revising it critically for important intellectual content. MN, TA, TH, YR, MM, TO1, and TO2 approved the final version to be published. Each author participated sufficiently in the work to take public responsibility for appropriate portions of the content and agreed to be accountable for all aspects of the work in ensuring that questions related to the accuracy or integrity of any part of the work are appropriately investigated and resolved. All authors read and approved the final manuscript.

\section{Acknowledgements}

The Authors thank the Department of Nutrition at Kanagawa Cancer Center for performing nutritional counselling and collecting nutritional data. The authors express their sincere gratitude to Ms. Natsumi Sato and Ms. Rika Takahashi for their excellent management of the data in this study.

\section{References}

1 Japanese Gastric Cancer Association: Japanese gastric cancer treatment guidelines 2014 (ver. 4). Gastric Cancer 20(1): 1-19, 2017. PMID: 27342689. DOI: 10.1007/s10120-016-0622-4

2 Waddell T, Verheij M, Allum W, Cunningham D, Cervantes A and Arnold D: Gastric cancer: ESMO-ESSO-ESTRO Clinical Practice Guidelines for diagnosis, treatment and follow-up. Radiother Oncol 110(1): 189-194, 2014. PMID: 24636158. DOI: 10.1016/j.radonc.2013.09.015

3 Yoshikawa T, Rino Y, Yukawa N, Oshima T, Tsuburaya A and Masuda M: Neoadjuvant chemotherapy for gastric cancer in Japan: a standing position by comparing with adjuvant chemotherapy. Surg Today 44(1): 11-21, 2014. PMID: 23508452. DOI: $10.1007 / \mathrm{s} 00595-013-0529-1$

4 Liedman B, Andersson H, Bosaeus I, Hugosson I and Lundell L: Changes in body composition after gastrectomy: results of a controlled, prospective clinical trial. World J Surg 21(4): 416-20; discussion 420-1, 1997. PMID: 9143575. DOI: 10.1007/p100012264

5 Fein M, Fuchs KH, Thalheimer A, Freys SM, Heimbucher J and Thiede A: Long-term benefits of Roux-en-Y pouch reconstruction after total gastrectomy: a randomized trial. Ann Surg 247(5): 759-765, 2008. PMID: 18438112. DOI: 10.1097/ SLA.0b013e318167748c

6 Lee SS, Yu W, Chung HY, Kwon OK and Lee WK: Using quality of life scales with nutritional relevance after gastrectomy: a challenge for providing personalized treatment. J Gastric Cancer 17(4): 342-353, 2017. PMID: 29302374. DOI: 10.5230/ jgc.2017.17.e39 
7 Aoyama T, Yoshikawa T, Shirai J, Hayashi T, Yamada T, Tsuchida K, Hasegawa S, Cho H, Yukawa N, Oshima T, Rino Y, Masuda $\mathrm{M}$ and Tsuburaya A: Body weight loss after surgery is an independent risk factor for continuation of $\mathrm{S}-1$ adjuvant chemotherapy for gastric cancer. Ann Surg Oncol 20(6): 20002006, 2013. PMID: 23242818. DOI: 10.1245/s10434-012-2776-6

8 Aoyama T, Kawabe T, Fujikawa H, Hayashi T, Yamada T, Tsuchida K, Yukawa N, Oshima T, Rino Y, Masuda M, Ogata $\mathrm{T}$, Cho $\mathrm{H}$ and Yoshikawa $\mathrm{T}$ : Loss of lean body mass as an independent risk factor for continuation of S-1 adjuvant chemotherapy for gastric cancer. Ann Surg Oncol 22(8): 25602566, 2015. PMID: 25515199. DOI: 10.1245/s10434-0144296-z

9 Iivonen MK, Koskinen MO, Ikonen TJ and Matikainen MJ: Emptying of the jejunal pouch and Roux-en-Y limb after total gastrectomy - a randomised, prospective study. Eur J Surg 165(8): 742-747, 1999. PMID: 10494638. DOI: 10.1080/ 11024159950189500

10 Takase M, Sumiyama Y and Nagao J: Quantitative evaluation of reconstruction methods after gastrectomy using a new type of examination: digestion and absorption test with stable isotope 13C-labeled lipid compound. Gastric Cancer 6(3): 134-141, 2003. PMID: 14520525. DOI: 10.1007/s10120-003-0238-3

11 An JY, Choi MG, Noh JH, Sohn TS, Jin DK and Kim S: Clinical significance of ghrelin concentration of plasma and tumor tissue in patients with gastric cancer. J Surg Res 143(2): 344-349, 2007. PMID: 17688884. DOI: 10.1016/j.jss.2007.02.017

12 Liedman B, Andersson H, Berglund B, Bosaeus I, Hugosson I, Olbe L and Lundell L: Food intake after gastrectomy for gastric carcinoma: the role of a gastric reservoir. Br J Surg 83(8): 11381143, 1996. PMID: 8869329. DOI: 10.1002/bjs.1800830835

13 Bae JM, Park JW, Yang HK and Kim JP: Nutritional status of gastric cancer patients after total gastrectomy. World J Surg 22(3): 254-60; discussion 260-1, 1998. PMID: 9494417. DOI: $10.1007 / \mathrm{s} 002689900379$

14 Watanabe M, Yamaoka K, Yokotsuka M, Adachi M and Tango T: Validity and reproducibility of the FFQ (FFQW82) for dietary assessment in female adolescents. Public Health Nutr 14(2): 297-305, 2011. PMID: 20537215. DOI: 10.1017/S13689800 10001618

15 Tabacchi G, Amodio E, Di Pasquale M, Bianco A, Jemni M and Mammina $\mathrm{C}$ : Validation and reproducibility of dietary assessment methods in adolescents: a systematic literature review. Public Health Nutr 17(12): 2700-2714, 2014. PMID: 24476625. DOI: $10.1017 / \mathrm{S} 1368980013003157$
16 Nakada K, Takahashi M, Ikeda M, Kinami S, Yoshida M, Uenosono Y, Kawashima Y, Nakao S, Oshio A, Suzukamo Y, Terashima M and Kodera Y: Factors affecting the quality of life of patients after gastrectomy as assessed using the newly developed PGSAS-45 scale: A nationwide multi-institutional study. World J Gastroenterol 22(40): 8978-8990, 2016. PMID: 27833389. DOI: $10.3748 /$ wjg.v22.i40.8978

17 Japanese Gastric Cancer Association: Japanese classification of gastric carcinoma: 3rd English edition. Gastric Cancer 14(2): 101-112, 2011. PMID: 21573743. DOI: 10.1007/s10120-0110041-5

18 Yamada T, Hayashi T, Cho H, Yoshikawa T, Taniguchi H, Fukushima $\mathrm{R}$ and Tsuburaya A: Usefulness of enhanced recovery after surgery protocol as compared with conventional perioperative care in gastric surgery. Gastric Cancer 15(1): 3441, 2012. PMID: 21573918. DOI: 10.1007/s10120-011-0057-x

19 Yamada T, Hayashi T, Aoyama T, Shirai J, Fujikawa H, Cho H, Yoshikawa T, Rino Y, Masuda M, Taniguchi H, Fukushima R and Tsuburaya A: Feasibility of enhanced recovery after surgery in gastric surgery: a retrospective study. BMC Surg 14: 41, 2014. PMID: 25001198. DOI: 10.1186/1471-2482-14-41

20 Lee HO, Han SR, Choi SI, Lee JJ, Kim SH, Ahn HS and Lim $\mathrm{H}$ : Effects of intensive nutrition education on nutritional status and quality of life among postgastrectomy patients. Ann Surg Treat Res 90(2): 79-88, 2016. PMID: 26878015. DOI: 10.4174/ astr.2016.90.2.79

21 Eom BW, Kim J, Kim DH, Kim YI, Yoon HM, Cho SJ, Lee JY, Kim CG, Choi IJ, Kim YW, Nam BH and Ryu KW: Recovery of food intake after gastrectomy for gastric cancer: based on a large-scale gastric cancer cohort. Dig Surg 35(3): 220-229, 2018. PMID: 28637034. DOI: 10.1159/000477779

22 Noguchi Y, Tsuburaya A, Makino T, Fukuzawa K, Nomura K, Yoshikawa T, Xu HM, Imada T and Matsumoto A: Metabolic alterations in totally gastrectomised patients-caloric intake and energy consumption. Asian J Surg 15(3): 97-102, 1992.
Received March 23, 2021

Revised April 9, 2021

Accepted April 14, 2021 